\title{
Numerical investigation on ballistic resistance of aluminium multi-layered panels impacted by improvised projectiles
}

Received: 29 December 2016 / Accepted: 24 March 2017 / Published online: 4 April 2017

(C) The Author(s) 2017. This article is an open access publication

\begin{abstract}
This paper presents a numerical study of perforation of the aluminium multi-layered targets impacted by steel projectiles in the shape of a ball, nut and nail. For the construction of the protective panels arc-, rectangular-, V- and U-shaped sheets of metal were considered. During the tests the panel thickness was constant at $160 \mathrm{~mm}$. The panels were composed of $1.5-\mathrm{mm}$-thick aluminium sheets, and the initial speed of debris was $500 \mathrm{~m} / \mathrm{s}$. A comprehensive numerical study indicated the shape of the layer that had superior ballistic resistance and the best strength-to-weight ratio. Computational analyses were also used to investigate the influence of thermal softening and strain rate hardening in the Johnson-Cook constitutive model on the ballistic performance of layered targets. The number of layers required to stop the penetrating objects was compared for the rigid and deformable projectiles. Based on the comparative studies, some guidelines for engineering tasks involving exploration of number of possible technical solutions were proposed.
\end{abstract}

Keywords Ballistic resistance $\cdot$ Multi-layered panels $\cdot$ Improvised projectiles

\section{Introduction}

The idea of using layered structures in ballistic resistant barriers is not new and has been extensively discussed in the literature. Designing protective panels places great emphasis on ensuring protection against punctures because the injuries from flying debris may carry significant risks for both civilians and military personnel. Previous studies were focused on ballistic resistance of single-layered targets and on potential improvements from replacing monolithic plates by multi-layered structures. However, there are not that many studies of more complex shapes of the component plates and of effectiveness of this solution with respect to ballistic perforation, especially by improvised projectiles.

The behaviour of multi-layered panels made of mild steel, stainless steel and aluminium plates under impact was studied in [1]. It was found that layered structures may offer better performance than monolithic plates due to change from plastic energy dissipation to perforation and stretching sheets of metal. Radin and Goldsmith [2] found that the ballistic resistance of a monolithic plate is superior to the structure of the same thickness but made of adjoined layers. It was also found that spaced layers usually displayed lower resistance

M. Szymczyk · W. Sumelka ( $\varangle) \cdot$ T. Łodygowski

Institute of Structural Engineering, Poznań University of Technology, Piotrowo 5 Street, 60-969 Poznań, Poland

E-mail: wojciech.sumelka@ put.poznan.pl

M. Szymczyk

E-mail: michal.g.szymczyk@ doctorate.put.poznan.pl

T. Łodygowski

E-mail: tomasz.lodygowski@put.poznan.pl 
than plates in contact. Almohandes et al. [3] conducted extensive experimental studies to determine the effects of target configuration on ballistic performance. Through many perforation tests it was established that single steel plates were more effective than multi-layered targets, with or without spacing. In the paper by Ben-Dor et al. [4] investigation of ballistic performance of layered targets was performed. They found that the width of the air gap between the layers had no effect on the resistance of target for the conical-nosed impactors. In the corresponding paper [5] it was concluded that the increase in the width of the air gaps and the increase in the number of layers raised the ballistic limit velocity of the target (understood as the velocity required for a projectile to penetrate a particular piece of material). The effect of air gaps upon ballistic properties of the shield was further discussed in [6], where the authors concluded that for the non-conical shape of the projectile changes in the ballistic limit velocity were in the range of several per cent. Woodward and Cimpoeru [7] investigated perforation of laminated and homogeneous plate targets by flat-ended and conical penetrators. The comparison of calculated and experimental ballistic limit showed that superior resistance occurred for the target system with two plates. In the work of Zukas and Scheffler [8] numerical results of the impact effect in monolithic and layered targets were presented. It was revealed that layering significantly weakened thin and intermediate thickness targets. Liang et al. [9] conducted studies of the resistance performance of multi-layered structures based on three conservation laws. They validated their results with the experimental data from Almohandes et al. [3]. Another comparative analysis of computational results and experimental data was presented by Elek et al. [10] where perforation of monolithic and layered targets was considered. They showed that for flat-ended cylindrical penetrator increasing the number of layers caused degradation of ballistic resistance of the target. Gupta et al. [11] presented experimental and numerical investigation of the ballistic performance of the layered aluminium plates under impact of flat-, ogive- and hemispherical-nosed projectiles. They showed that residual velocity of the projectile was similar for targets of equivalent thickness with one or two layers. Also, the ogive-nosed projectile was found to be the most efficient for multi-layered panel. Dey et al. [12] investigated perforation resistance of double-layered steel plates impacted by blunt and ogival projectiles. They found that double layering of the target increased the ballistic limit velocity for spaced plates by nearly $40 \%$ compared to a monolithic target of equal thickness. In contrast, based on numerical investigation of monolithic, double- and triple-layered plates Flores-Johnson et al. [13] concluded that monolithic plates had a better ballistic performance than a layered target. Multi-layered structures in different combinations were also considered in research conducted by Deng et al. [14]. Results of an experimental test of panels with one, two, three or four plates showed that monolithic targets had higher ballistic resistance than layered targets if the total thickness was smaller than the value where petaling or bulging becomes the dominant failure mode. Finally, Ben-Dor et al. [15] presented a comprehensive survey of experimental and numerical investigations of multi-layered targets and also delivered a review of analytical methods for optimization of layered panels.

It is crucial to use a material model that can mimic a mechanical response that is in good agreement with the behaviour of a physical target. In this study, the computational model is based on the constitutive model proposed by Johnson and Cook [16,17] — the one which assures good global damage approximation (cf. $[18,19])$, thus appropriate for herein considerations. Since the time it was published, a number of modifications have been proposed but the original formulation is still used for research of perforation [20]. It should be emphasized that the same class of models was developed earlier by Litoński in [21] where the torsion of tubes made of strain rate-sensitive plastic material was examined. Li et al. [22] proposed a modified Johnson-Cook model that can provide better coupling for the influence of temperature and strain rate on flow behaviour. The proposed model showed good ability to track the flow behaviour of T24 steel over the entire temperature and strain rate range. A modified version of the Johnson-Cook constitutive relation (the MJC model) was used in research conducted by Børvik et al. [23], where perforation of five different steel plates was investigated. Material constants for the steel plates were based on various material tests, and the data for bullets were mainly taken form the literature. Samantaray et al. [24] performed a comparative study of Johnson-Cook, modified Zerilli-Armstrong and Arrhenius-type constitutive models. The experimental data from compressive tests were used to establish material constants for each of those formulations. In contrast to the previous works it was found that the Johnson-Cook model was incapable of providing a good description of flow behaviour of $9 \mathrm{Cr}-1 \mathrm{Mo}$ steel over the entire range of strain rates, temperatures and strains. The significance of the use of strain rate and temperature parameters in the model was confirmed in the work presented by B $\varnothing$ rvik et al. [25]. The fully coupled model where thermal and strain rate effects were included showed best agreement between experimental and numerical results. A comprehensive study of the extruded aluminium panels was presented by Børvik et al. [26]. The experimental results were used to calibrate a modified version of the Johnson-Cook constitutive relation and failure mode. This computational effort delivered a very good agreement between numerical and experimental data since the mechanical behaviour of the target was captured. 
In the corresponding study by Holmen et al. [27] perforation of empty and sand-filled aluminium panels was investigated. It was shown that though the combination of the finite element analysis (Johnson-Cook plasticity and failure models) and the discrete particle method can be used to describe interaction between solid and granular media, still some challenges remain.

In the work done by Corran et al. [1] it was shown that the nose shape, in particular the nose radius, was extremely important in regard to likelihood of the target penetration. Wingrove [28] found that flatended projectiles penetrate the target with less resistance than spherical- and ogive-shaped impactors. This phenomenon was attributed to the change in failure mode from adiabatic shear zones for the blunt projectiles, to the plastic tearing for the spherical- and ogive-shaped objects. Wilkins [29] showed that the minimum ballistic velocity was lower for a sharp projectile perforating thick targets than for a blunt projectile. In case of thin targets, an opposite dependence was found. In papers [30] and [31] perforation of steel plates by three different projectiles was examined. Both experimental and computational experiments showed that hemispherical and conical shaped impactors gave ballistic limit velocity close to $300 \mathrm{~m} / \mathrm{s}$, while for the blunt projectile this parameter was about $185 \mathrm{~m} / \mathrm{s}$. Furthermore, it was stated that at the highest impact velocities, deformation of the projectile might become a significant factor and might influence the overall resistance of the panel. Gupta et al. [32] presented experimental and numerical investigations of perforation of thin aluminium plates by blunt and hemispherical-nosed projectiles. The results obtained in that study were similar to those previously discussed; namely higher ballistic limit was found for the hemispherical projectile. In the investigation by Arias et al. [33] good agreement between experimental and numerical results was obtained for the cylindrical, conical and hemispherical projectiles. It was shown that residual velocity after perforation was similar for a conical- and a hemispherical-shaped object, while the lowest value was found for a cylindrical projectile. Visible correlation between the projectile shape and the energy absorption capacity of a target was presented in the paper by Kpenyigba et al. [34]. It was found that the hemispherical-nosed impactor was the least efficient, followed by conical and two double nosed. In the work by Rusinek et al. [35] primary focus was on studying the conical projectile diameter in relation to its perforation performance. Numerical and experimental analysis revealed that a linear relation existed between ballistic limit velocity and projectile diameter. In the paper by Iqbal et al. [36] the influence of the target-to-projectile diameter ratio D/d on the ballistic resistance and failure mechanism was investigated. For the blunt-nosed projectile the increase in the ballistic limit velocity was observed up to the point where $\mathrm{D} / \mathrm{d}$ was equal to 10 ; after that the change in the value of ballistic limit velocity was negligible. For the ogive-nosed projectile the value of limit velocity was increasing up to the point where D/d was 25; after that, the relation between ballistic limit velocity and the target-to-projectile ratio was not observed.

The influence of strain rate and temperature on material parameters was investigated by Klepaczko [37] and formulated into a power law constitutive relation. In [38] four constitutive models for metals were researched in terms of the work hardening limitations. It was concluded that the original form of Johnson-Cook model may be inadequate for describing the behaviour of metals that exhibit inversely proportional relation between work hardening and strain rate. In the work by Rusinek et al. [39] energy absorption properties of a target under various strain rates were examined. It was observed that the work hardening of the analysed material (AISI 304 steel) was independent of plastic strain. Pothnis et al. [40] found that for both aluminium alloy 7075 T651 and IS 2062 mild steel tensile yield strength was enhanced when different strain rates were applied, if compared to quasi-static loading. Hor et al. [41] conducted a number of compression and shear tests involving a wide range of temperatures $\left(20-1000^{\circ} \mathrm{C}\right)$ and strain rates $\left(10^{-2}-10^{5} \mathrm{1} / \mathrm{s}\right)$. They found strong coupling of three main phenomena: effect of the temperature, effect of the strain rate and the strain hardening-softening. Thus, the material model that includes those effects was required in case of dynamic loading. In [42] the dynamic mechanical response of Fe-based high-temperature alloy over a wide range of strain rates and temperatures was investigated. The effects of strain rate hardening and thermal softening were visible in the presented data and were the base for the formulation of power law constitutive model.

One of the goal of this study is to provide a set of guidelines for those who are interested in the properties of computational models of spaced multi-layered targets (composed of thin metallic sheets) perforated by the improvised impactors. We have examined the behaviour of layered structures including or excluding thermal and strain rate effects in various configurations. Rigid and deformable projectiles were also used to establish how these constraints translated to the ballistic resistance of the panel. The ballistic performance of numerical models of nail-, nut- and ball-shaped impactors in different orientations was examined. Furthermore, ballistic performance of four different shapes of the component layers was tested against projectiles in three different points of impact. 
This paper extends previous research of the authors regarding perforation of multi-layered panels by rigid projectiles [43]. Here, the results include the study of the impact of deformable projectiles, and new shapes of panel layers are considered as well. Moreover, different combinations of the work hardening and thermal softening effects in the computational model and their impact on mechanical response of the layered panel are examined.

\section{Mathematical modelling}

\subsection{Fundamental concepts}

The Clausius-Duhem inequality is stated in the form

$$
\sigma: \mathbf{d}-\rho(\dot{\psi}+\eta \dot{\vartheta})-\frac{1}{\vartheta} \mathbf{q} \cdot \operatorname{grad} \vartheta \geq 0,
$$

where $\sigma$ is the Cauchy stress, $\mathbf{d}$ is the symmetric part of the velocity gradient, $\rho$ is the current density, $\psi=e-\vartheta \eta$ denotes the free energy function, $e$ is the specific internal energy, $\vartheta$ is the temperature, $\eta$ is the specific entropy, and $\mathbf{q}$ is the heat flux. Under the assumption that the free energy function has the form

$$
\psi=\psi(\mathbf{F}, \vartheta, \alpha)
$$

where $\mathbf{F}$ is the deformation gradient, and $\alpha$ is a set of $k$-th internal variables, Eq. (1) obtains the form

$$
\left(\sigma \cdot \mathbf{F}^{-T}-\rho \frac{\partial \psi}{\partial \mathbf{F}}\right): \dot{\mathbf{F}}-\rho\left(\eta+\frac{\partial \psi}{\partial \vartheta}\right) \dot{\vartheta}-\rho \frac{\partial \psi}{\partial \alpha_{k}} * \dot{\alpha_{k}}-\frac{1}{\vartheta} \mathbf{q} \cdot \operatorname{grad} \vartheta \geq 0,
$$

where $(*)$ denotes the appropriate product operation. For an adiabatic process, assuming the additive decomposition of the total strain into the elastic part and plastic part, namely

$$
\varepsilon=\varepsilon^{e}+\varepsilon^{p}
$$

the free energy function takes the form

$$
\psi=\psi\left(\varepsilon, \varepsilon^{p}, \bar{\varepsilon}^{p}\right)=\psi^{e}\left(\varepsilon-\varepsilon^{p}\right)+\psi^{p}\left(\bar{\varepsilon}^{p}\right),
$$

and Eq. (3) can be rewritten as

$$
\left(\sigma-\bar{\rho} \frac{\partial \psi^{e}}{\partial \varepsilon^{e}}\right): \dot{\varepsilon}^{e}+\sigma: \dot{\varepsilon}^{p}-\bar{\rho} \frac{\partial \psi^{p}}{\partial \bar{\varepsilon}^{p}} \dot{\bar{\varepsilon}}^{p} \geq 0,
$$

where $\bar{\rho}$ is a reference density, $\alpha=\left\{\bar{\varepsilon}^{p}\right\}$, and $\bar{\varepsilon} p$ denotes the equivalent plastic strain. Therefore, the elastic stress relation is

$$
\sigma=\bar{\rho} \frac{\partial \psi^{e}}{\partial \varepsilon^{e}}
$$

and the evolution of temperature reduces to

$$
\bar{\rho} c_{p} \dot{\vartheta}=\chi \sigma: \dot{\varepsilon}^{p}
$$

where $c_{p}$ denotes the specific heat, $\chi$ is the irreversibility coefficient, and assuming isotropy of the elastic range we have $\bar{\rho} \psi^{e}=\frac{1}{2} \varepsilon^{e}: \mathcal{L}^{e}: \varepsilon^{e}$, where $\mathcal{L}^{e}$ is the standard isotropic elasticity tensor.

Finally, the evolution of plastic strain is assumed in the form

$$
\dot{\varepsilon}^{p}=\dot{\lambda} \frac{\partial f}{\partial \sigma},
$$

where $\lambda$ denotes the intensity of the plastic flow, and $f$ denotes the Huber-Mises-Hencky yield surface. For Eq. (9) the following constraints apply, which define when the evolution of plastic strain and internal variables $(\dot{\lambda} \neq 0)$ may occur:

$$
f-\sigma_{y}\left(\bar{\varepsilon}^{p}\right) \leq 0, \quad \dot{\lambda}>0, \quad \dot{\lambda}\left(f-\sigma_{y}\left(\bar{\varepsilon}^{p}\right)\right)=0,
$$

where $\sigma_{y}$ denotes the yield stress. 
Table 1 List of material parameters and mechanical properties of the steel and the aluminium [44,45]

\begin{tabular}{lll}
\hline Parameters & Material & \\
\cline { 2 - 3 } & Aluminium & Steel \\
\hline$A(\mathrm{MPa})$ & 324 & 640 \\
$B(\mathrm{MPa})$ & 113 & 113 \\
$n(-)$ & 0.42 & 0.42 \\
$m(-)$ & 1.34 & 1.34 \\
$\vartheta_{\text {melt }}\left({ }^{\circ} \mathrm{C}\right)$ & 925 & 1200 \\
$\vartheta_{\text {transition }}\left({ }^{\circ} \mathrm{C}\right)$ & 293.2 & 293.2 \\
$\bar{\rho}\left(\mathrm{kg} / \mathrm{m}^{3}\right)$ & 2703 & 7850 \\
Young's modulus $(\mathrm{GPa})$ & 69 & 209 \\
Poisson's ratio $(-)$ & 0.3 & 0.3 \\
$C(-)$ & 0.002 & 0.002 \\
$\dot{\varepsilon_{0}}\left(1 / \mathrm{s}{ }^{-1}\right)$ & 1 & 1 \\
$c_{p}(\mathrm{~J} / \mathrm{kg} \cdot \mathrm{K})$ & 910 & 460 \\
$\chi(-)$ & 0.9 & 0.9 \\
\hline
\end{tabular}

\subsection{Evolution function for yield stress}

As mentioned earlier, the Johnson-Cook model was chosen to describe the evolution of the yield stress for both aluminium target and steel impactor. The model takes into account the strain rate hardening and the thermal softening effects [16]. The yield stress $\sigma_{y}$ can be written as

$$
\sigma_{y}=\left[A+B\left(\bar{\varepsilon}^{\mathrm{pl}}\right)^{n}\right]\left[1+C \ln \left(\frac{\dot{\bar{\varepsilon}}^{\mathrm{pl}}}{\dot{\varepsilon}_{0}}\right)\right]\left(1-\hat{\vartheta}^{m}\right),
$$

where $A, B, m$ and $n$ are the material parameters measured at temperature lower than the transition temperature, $C$ is a dimensionless strain rate hardening coefficient, $\bar{\varepsilon}^{\mathrm{pl}}$ is an equivalent plastic strain, $\dot{\varepsilon}_{0}$ is a reference rate of strain, and $\hat{\vartheta}$, a dimensionless temperature parameter, is defined as

$$
\hat{\vartheta} \equiv \begin{cases}0 & \text { for } \vartheta<\vartheta_{\text {transition }} \\ \left(\vartheta-\vartheta_{\text {transition }}\right) /\left(\vartheta_{\text {melt }}-\vartheta_{\text {transition }}\right) & \text { for } \vartheta_{\text {transition }} \leq \vartheta \leq \vartheta_{\text {melt }} \\ 1 & \text { for } \vartheta>\vartheta_{\text {melt }}\end{cases}
$$

where $\vartheta_{\text {melt }}$ is the melting temperature of metal and $\vartheta_{\text {transition }}$ denotes the limit below which the yield stress in Eq. (11) is not temperature dependent. The parameters required for the proper implementation of the JohnsonCook model are given in Table 1.

\subsection{Damage initiation and fracture conditions}

The dynamic failure model for the Johnson-Cook plasticity supplied by Abaqus was used [17]. It assumes that a fracture is initiated when the accumulated damage parameter $\omega$ exceeds 1 . The damage parameter $\omega$ is defined as

$$
\omega=\sum\left(\frac{\Delta \bar{\varepsilon}^{\mathrm{pl}}}{\bar{\varepsilon}_{D}^{\mathrm{pl}}}\right)
$$

where $\Delta \bar{\varepsilon}^{\mathrm{pl}}$ is an increment of the equivalent plastic strain, $\bar{\varepsilon}_{D}^{\mathrm{pl}}$ is the strain at the moment the damage is initiated, and the summation is performed over all increments in the analysis. The strain at failure $\left(\bar{\varepsilon}_{f}^{\mathrm{pl}}\right)$ depends on the dimensionless stress ratio $\eta$, dimensionless plastic strain rate $\dot{\bar{\varepsilon}}^{\mathrm{pl}} / \dot{\varepsilon}_{0}$ and the previously mentioned temperature parameter $\hat{\vartheta}$ :

$$
\bar{\varepsilon}_{D}^{\mathrm{pl}}=\left[d_{1}+d_{2} \exp \left(-d_{3} \eta\right)\right]\left[1+d_{4} \ln \left(\frac{\dot{\bar{\varepsilon}}^{\mathrm{pl}}}{\dot{\varepsilon}_{0}}\right)\right]\left(1+d_{5} \hat{\vartheta}\right)
$$

Stress triaxiality $\eta$ is given as a ratio of pressure stress $p$ and von Mises equivalent stress $\bar{\sigma}$. The parameters for the Johnson-Cook failure model used in perforation analysis are presented in Table 2. 
Table 2 Input parameters for the Johnson-Cook dynamic failure model [44]

\begin{tabular}{lll}
\hline Parameters & Material & Steel \\
\cline { 2 - 3 } & Aluminium & 3 \\
\hline$d_{1}(-)$ & -0.77 & 0 \\
$d_{2}(-)$ & 1.45 & 0 \\
$d_{3}(-)$ & 0.47 & 0 \\
$d_{4}(-)$ & 0.00314 & 0 \\
$d_{5}(-)$ & 1.6 & 0 \\
\hline
\end{tabular}
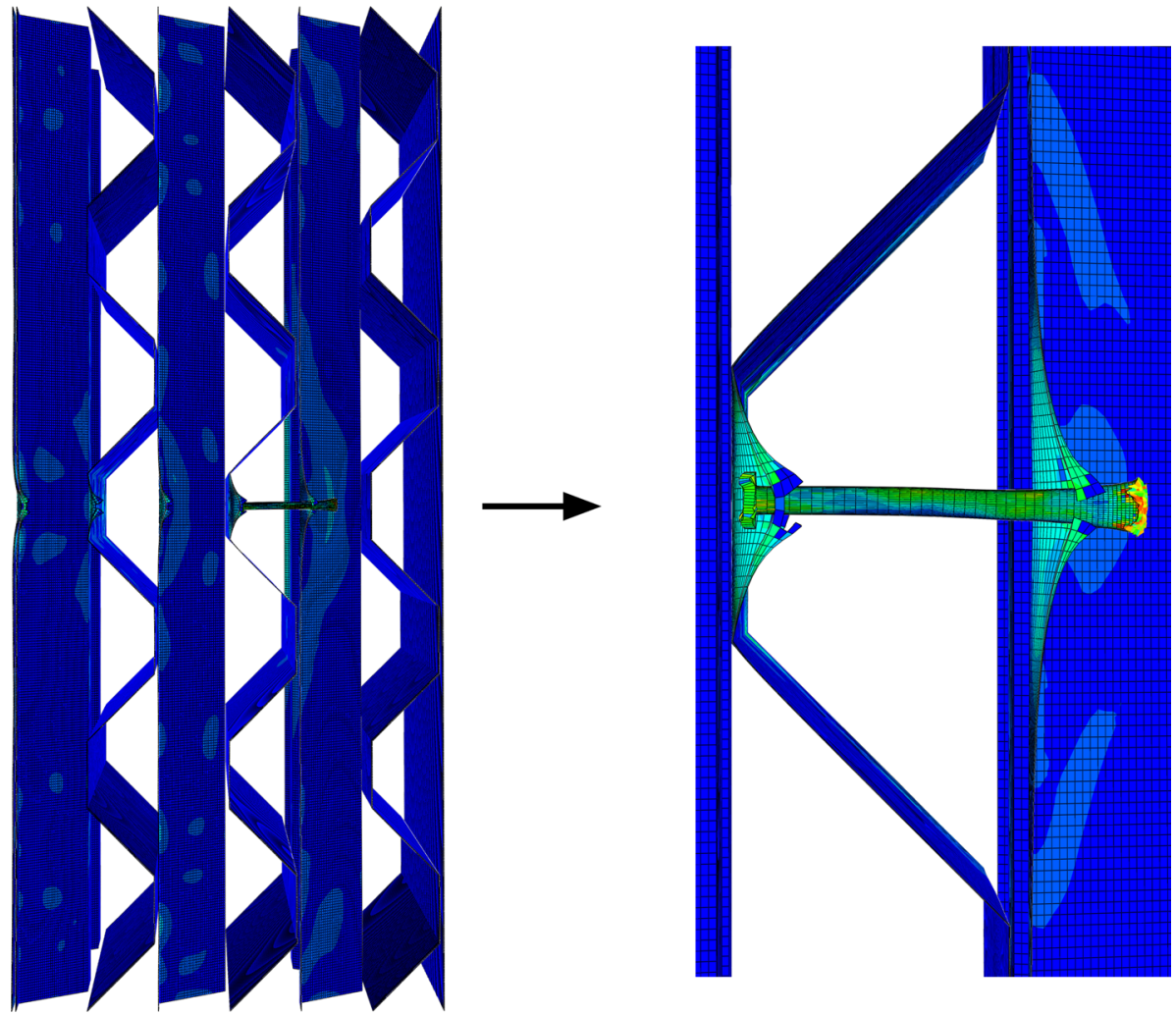

Fig. 1 Numerical model of the multi-layered panel and the projectile

In the present study, when the failure initiation criterion was met, the stress components were set to zero (in each Gauss point), which is equivalent to removing an element from the mesh. This behaviour was imposed by setting the fracture energy parameter to zero.

The projectile was additionally modelled as a rigid body for the purpose of certain analyses as mentioned. In those cases steel density was the only property required by the FE solver to calculate the projectile behaviour. Hard contact formulation was used to define the pressure-clearance relationship.

\section{Description of the numerical experiments}

The main tool used in our research is the numerical model of the multi-layered panel and the projectile which was subjected to many perforation tests. An example of a perforation test is presented in Fig. 1. The speed of the projectile and its current position relative to the initial position were recorded throughout the simulation. The objects were modelled in the three-dimensional space; hence, these parameters were tracked for three axes. The analysis was iterative, which means that the simulation started with an initial number of layers in the panel and if the object was not stopped by the panel (i.e., at the end of the analysis it had a non-zero speed), the number of layers was increased and the analysis repeated. The basic analysis time was set at $9.6 \times 10^{-4} \mathrm{~s}$ and was modified if necessary. 


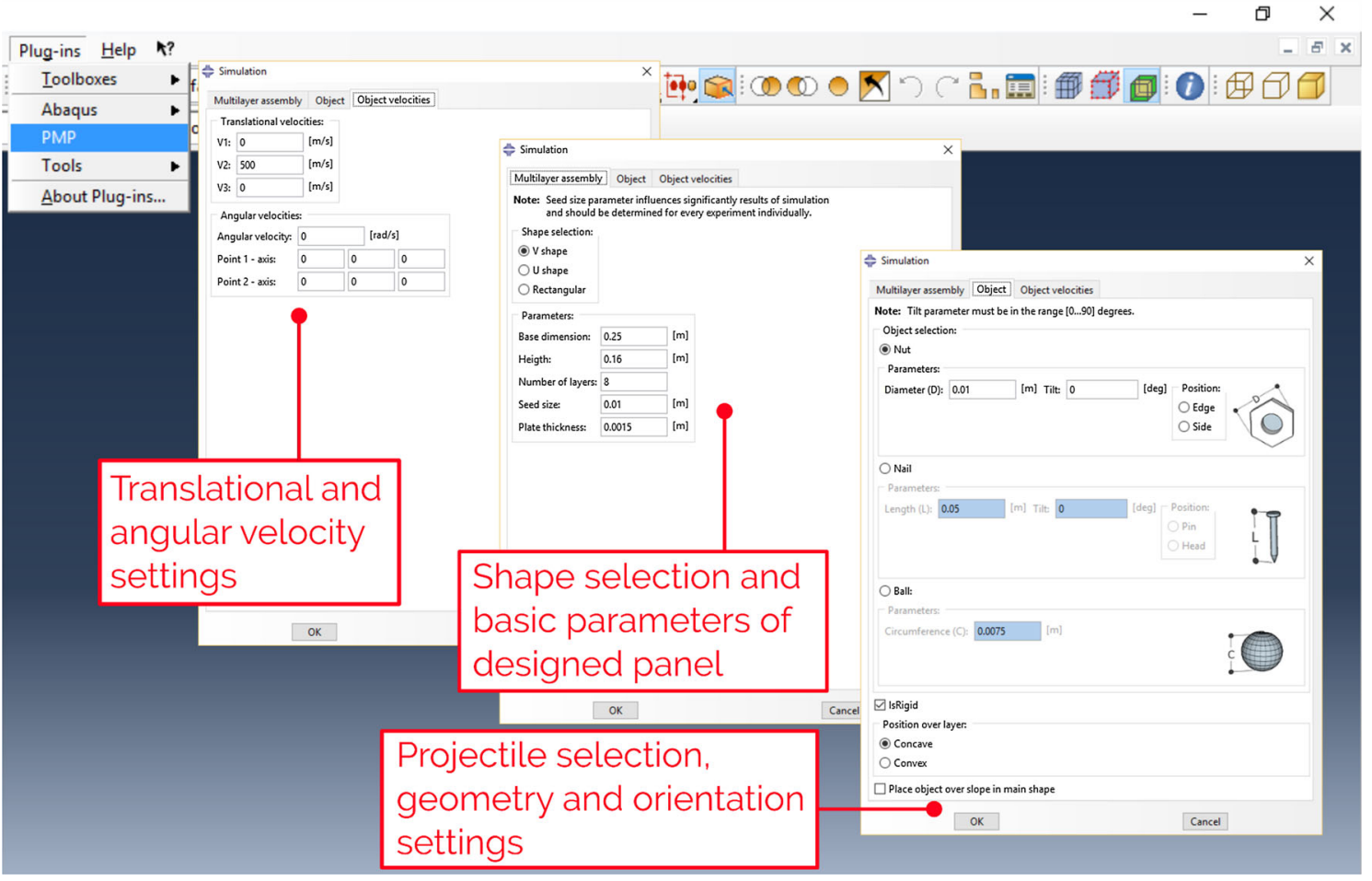

Fig. 2 Main tabs of the PMP plug-in

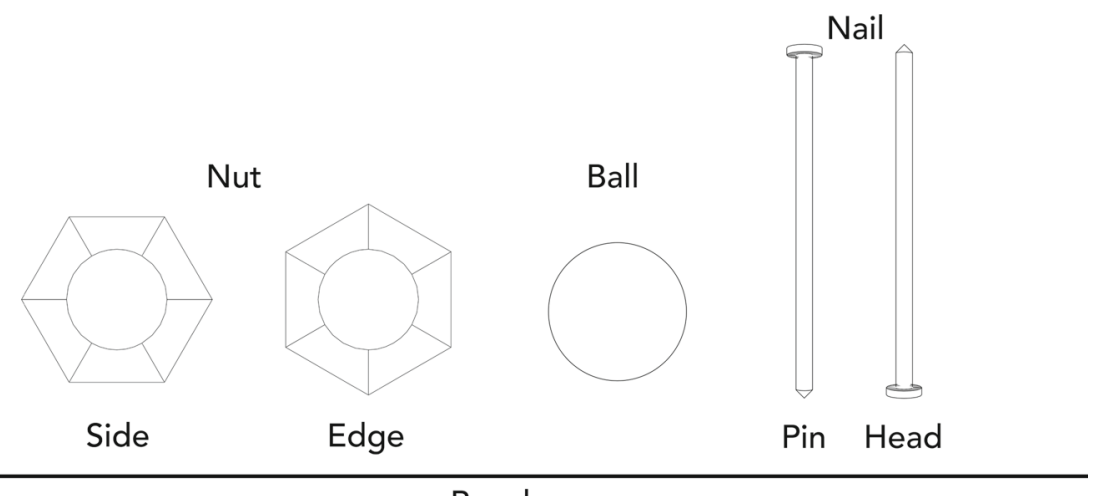

Panel

Fig. 3 Projectiles shapes and orientation with respect to the panel

The model was created and examined in the environment of Abaqus/Explicit software. For the purpose of efficient computation of many hundreds cases a specialized plug-in (called PMP) was developed which enabled fast assembly of the panel, as well as the setting of required parameters (Fig. 2). This software suite also has a powerful scripting mechanism which was extensively used in the course of our research. The computational model generated with the PMP plug-in allowed setting the tilt (the angle between the projectile and the outermost layer) and angular speed of the projectile; hence, the usage of model symmetry reduction was impossible.

In this study we considered three shapes of the projectile: nail, nut and ball, as depicted in Fig. 3. For the first two various orientations in space at the time of impact were taken into account (Fig. 3). It was assumed that either the nail head or pin can be directed towards the panel. The same reasoning was extended to the nut: both the edge and side positions were included in the simulations. As it was previously mentioned, both a rigid model and a deformable model of the projectile were examined. The dimensions were selected so that despite different shapes the objects had similar masses, approximately $2 \mathrm{~g}$. The initial velocity was fixed and equal to $500 \mathrm{~m} / \mathrm{s}$. 
(a)

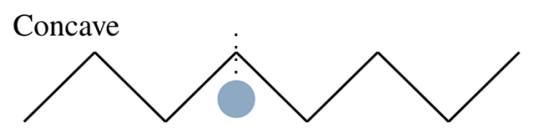

Convex

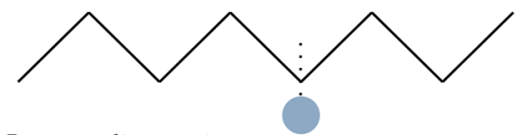

Intermediate point

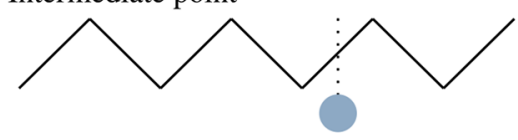

(b)

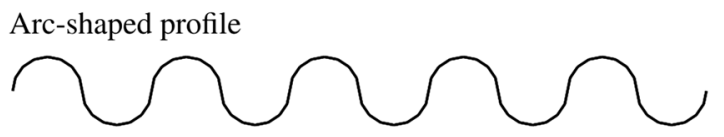

Rectangular-shaped profile

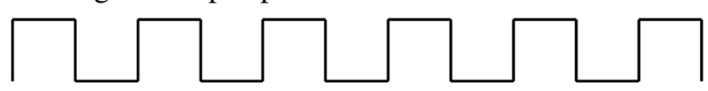

V-shaped profile

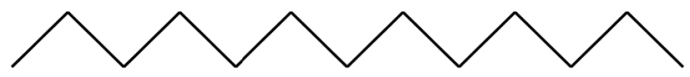

U-shaped profile

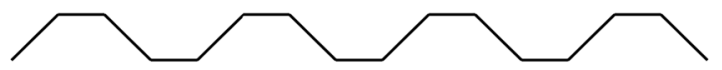

Plane sheet of aluminium

Fig. 4 A projectile's position relative to the panel (a) and profile shapes (b)

In our research we examined properties of barriers built with plane sheets of metal and also more complicated shapes, as shown in Fig. 4b. Therefore, for some panels more than one initial position of the projectile had to be included in the simulations. For every non-flat plate three different places of impact were identified: concave, convex and intermediate between these two-see Fig. 4a. Every panel used in the simulations was rectangular cuboid with a square base that was $0.25 \times 0.25 \mathrm{~m}^{2}$ in size and had a casing made of two additional flat plates placed at the front and back of the multi-layered structure. The thickness of the panel was $0.16 \mathrm{~m}$ and was constant, while the thickness of a single layer was variable and changed with the number of layers. The thickness of aluminium plates used in this study was $1.5 \mathrm{~mm}$. The basic dimensions were chosen to ensure an adequate distance from the place of impact to the edges of barriers; thus, boundary effects were eliminated.

Aluminium layers were modelled with S4R (shell, 4-node, reduced integration) elements, whereas C3D8R (continuum, 3-D, 8-node, reduced integration) elements were used for modelling the projectile. The maximum number of elements in the FE mesh was $\sim 1.3 \times 10^{6}$.

\section{Results}

In the numerical analysis the material model is a key element that significantly influences the outcome. In general, the more accurate the model, the closer to the behaviour of the real object is the behaviour of the tested structure. Introducing thermal and rate effects to the basic Johnson-Cook formula increased the complexity and the number of model parameters but did not yield better results in terms of engineering significance.

In the first part of the study we examined the relation between thermal and strain rate effects and the behaviour of the panel made of metal plates. Plane sheets were chosen since any profile could compromise the results by changing the trajectory of the projectile. The different perforating objects (ball, nut and nail) and different impact orientations assured that our research was independent of the shape of the projectile. At this stage the impactors were modelled as rigid bodies. The impact of the previously mentioned phenomena was assessed by recording the number of layers required to stop the impactor.

The results of attempts of the projectile to break through the panel are shown in Fig. 5. The results are divided into three separate groups. The first group in Fig. 5a concerns the simulations without thermal softening or strain hardening, the second group in Fig. 5b presents the results for the analysis with strain hardening (but no thermal dependence), and the third, plotted in Fig. 5c, shows results for thermal softening (without strain hardening). Figure 5 also shows how the weight of the panel changes with the number of layers. We can see that for the nut and ball the number of layers required to prevent the penetration is constant and independent of the effects included in the numerical model. The nail is the only projectile that changed the number of layers required to stop the perforation. Therefore, the nail was chosen for further study of the impact of thermal softening and strain hardening on the ballistic resistance of the target.

As shown above, changes in the model parameters are not necessarily reflected in the behaviour of multilayer structures for the selected projectiles. As in the first part of the study rigid impactors were used, we went 


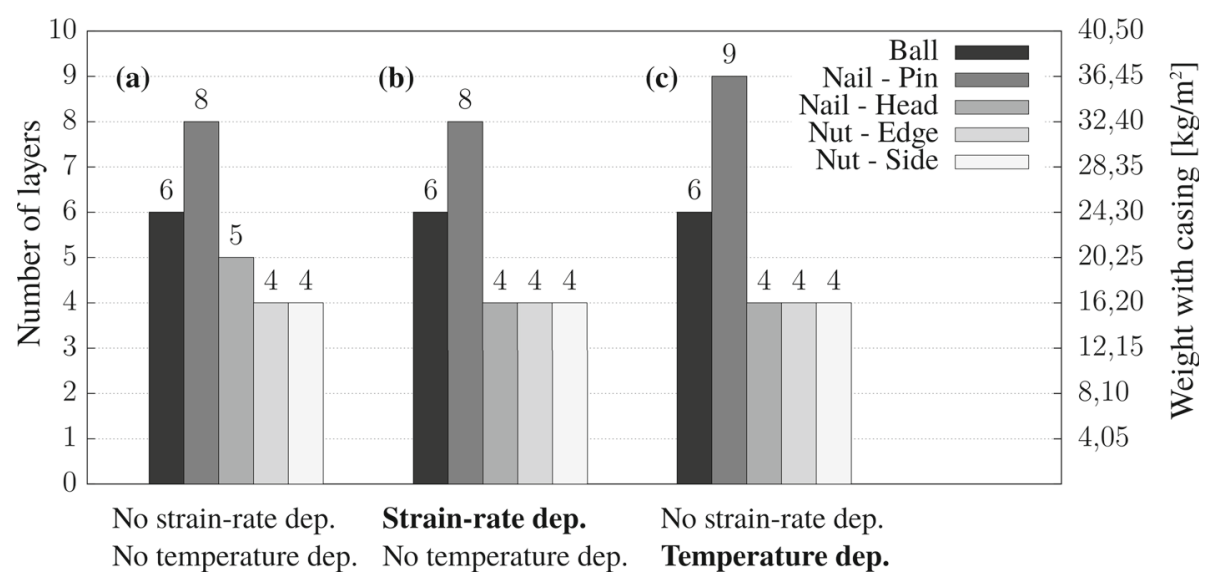

Fig. 5 Influence of thermal softening and strain hardening on the strength of the panel

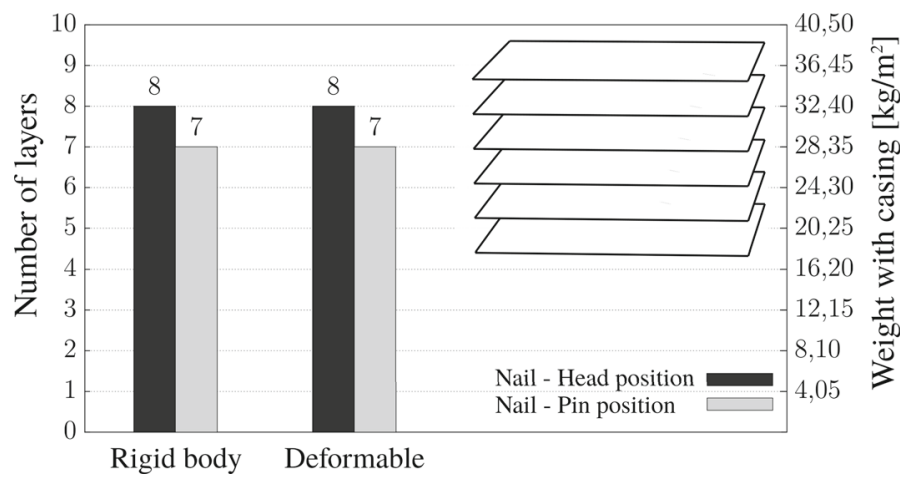

Fig. 6 Perforation results for the aluminium panel—material model with strain rate and thermal dependence

on to investigate whether the deformable projectile would trigger any change in the results. Figure 6 presents the perforation simulations with rigid and deformable objects; both thermal softening and strain hardening were included in the material model. Rigid and deformable projectiles require the same number of layers for the pin and head orientation. On the other hand, we noted a significant increase in the time needed to perform the calculations; it will be discussed later in this section.

Comparison of different shapes revealed that only the nail is worth investigating further (Fig. 5). Moreover, the mechanical model of the projectile does not introduce any noticeable change (Fig. 6). In other words, a rigid impactor is stopped by the panel of the same design, which is sufficient to stop the deformable object. This last conclusion was unexpected regarding our previous experience; so it was be developed in the following (second) part.

In the first series of numerical studies the barriers were built with flat plates. In the second series we focused on more complex shapes. On the one hand this allowed us to observe the differences in the behaviour of the deformable and rigid impactors and review the ability of these shapes to dissipate the energy of the projectile. Because the cross section of the layer was now more complex, we had to consider different piercing points, as mentioned earlier. Previous analysis had shown that the nail, particularly when the pin was directed towards the panel, was an effective tool for testing multi-layered structures. The aluminium model included rate and thermal dependence. The panels investigated in this part were closed with two additional sheets of aluminium, forming a uniform casing for the target, as mentioned before.

We notice that eight layers were required when the projectile punctured the concave part of the arc-shaped panel (cf. Fig. 7a). In this layer arrangement the same number of layers is effective only for the case of convex position. The intermediate point was recognized as a spot where the difference in projectile behaviour was the greatest. The rectangular shape (Fig. 7b) was a much more stable solution as an equal number of layers for the rigid and deformable objects was sufficient to prevent perforation. Again, the biggest difference in the behaviour of different models of projectiles can be observed in the intermediate point. For both of these cases the difference exists because in the intermediate position the impactor had to perforate additional material, 

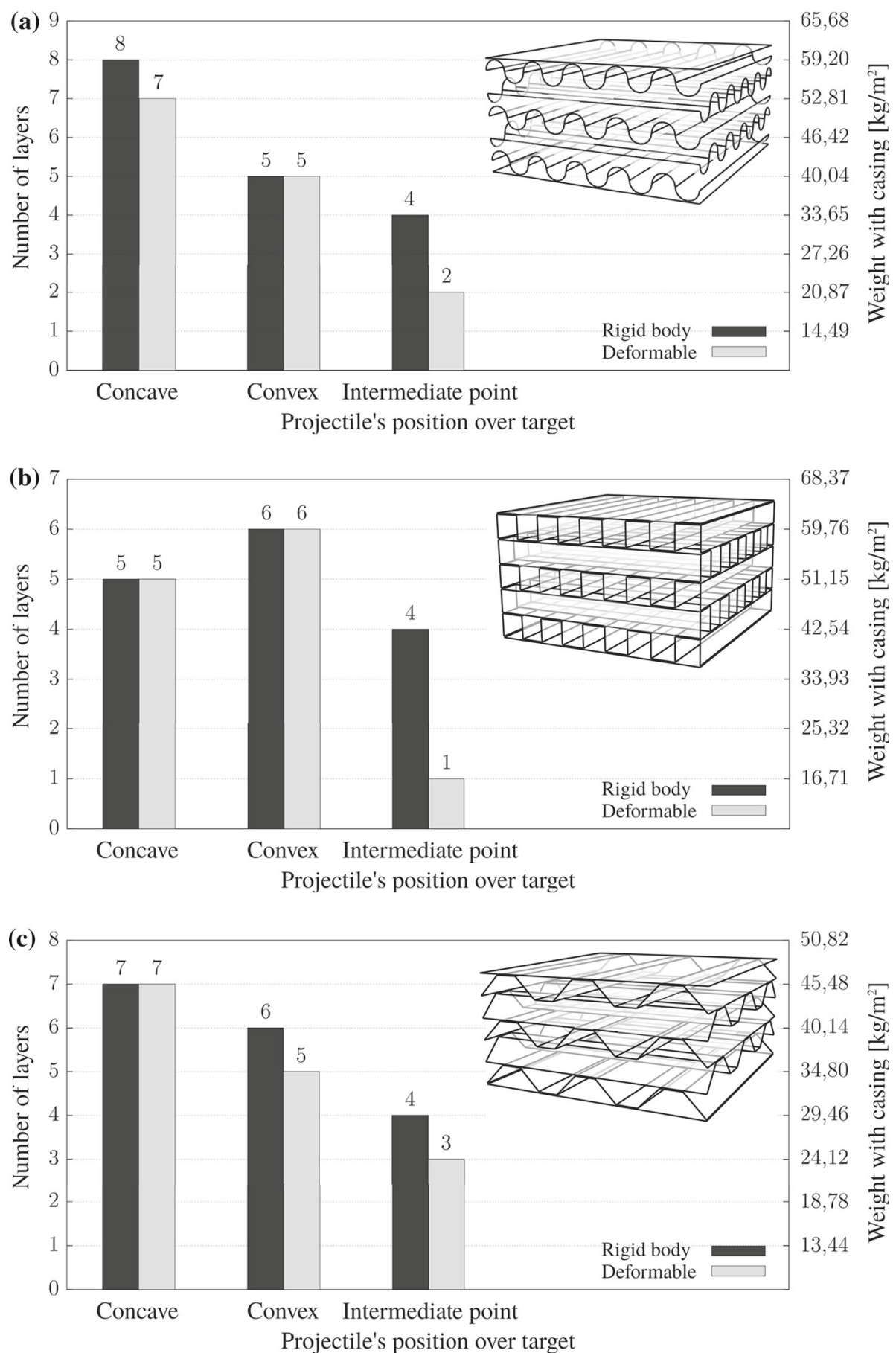

Fig. 7 Perforation results for the panel made of differently-shaped layers a arc-shaped panel b rectangular-shaped panel c U-shaped panel d V-shaped panel

which caused significant deformation of the projectile, resulting in considerable loss of its velocity. The same phenomena occurred in the convex point of the V-shaped panel but with less intensity, as there is only two-layer gap-see Fig. 7d. In other cases the perforation by the rigid or deformable objects had the same intensity in a specific position. The barrier with the U-shaped layers required seven layers to stop the projectile if it hits the concave part. Moreover, it was the only case where there was a difference between the results for the rigid and deformable objects. 


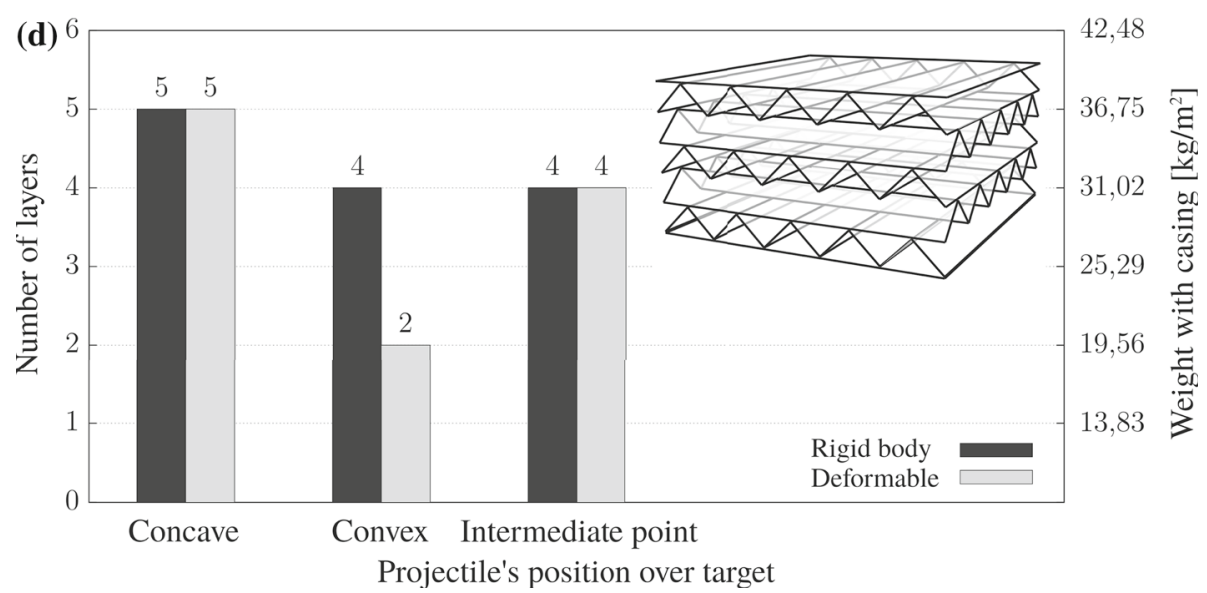

Fig. 7 continued

Table 3 Simulation time depending on the shape of the aluminium plates

\begin{tabular}{lll}
\hline Shape of the sheet & Rigid body & Deformable \\
\hline Arc shape & $00: 52: 00$ & $18: 59: 00$ \\
U shape & $01: 29: 00$ & $18: 16: 00$ \\
V shape & $01: 00: 00$ & $25: 51: 00$ \\
Rectangular shape & $01: 01: 00$ & $20: 04: 00$ \\
& (hh:mm:ss) & (hh:mm:ss) \\
\hline
\end{tabular}

In conclusion, it can be noticed that in general the concave part of panel is the weakest one; if the projectile hits this particular spot, the biggest number of layers was required to stop it. However, for the rectangularshaped panel the worst-case scenario occurred in the convex part (Fig. 7b). Therefore, in the search for the detail solution one should investigate different points of impact. The rectangular-shaped panel also provided the biggest difference in the behaviour of rigid and deformable projectiles. This difference can be attributed to the amount of aluminium that the projectile encountered puncturing this particular part; as a result, the deformations of the projectile were significant. Finally, the V-shaped panel with five layers is an optimal solution for stopping fast-moving debris. It can provide required protection and the best strength-to-weight ratio. It is worth noting that in six out of twelve results presented in Fig. 7 the rigid projectile required more layers than the deformable one. Application of a rigid projectile places us on the safe side even if the final design may be oversized. Because of this it is possible to omit the thermal effect and strain rate hardening while drafting the first prototype of the panel constructed of thin metallic layers.

The simulations were carried out on the Intel 64-bit PC (CPU 8x2.33 GHz Inter(R) Xeon(R), 16 GB RAM, OS GNU Linux). As it was mentioned earlier, the simulation time can increase significantly for deformable projectiles, as shown in Table 3. This is due to the increase in computational complexity resulting from the deformation of the projectile and creation of a new contact surface due to erosion, two factors which were not present in analysis of a rigid body behaviour. In some cases it can take 25 times longer to finish calculations for the same set-up with a different model of impactor.

It is worth noting that erosion of deformable objects requires defining the interior surface, which is not yet supported in the Abaqus/CAE. Therefore, first a model with the exterior surface has to be created and then changes have to be introduced in the resulting input file using the keyword INTERIOR.

\section{Conclusion}

Based on a significant number of numerical simulations the ballistic perforation of multi-layered targets impacted by improvised projectiles has been investigated. The following major conclusions can be stated:

- among various shapes of the component layers tested the V-shaped fulfilment of the panel was found to have the best strength-to-weight ratio,

- the impact position of the projectile was significant for the ballistic resistance of the target, 
- the shape of the projectile had a strong impact on the number of layers required to stop the perforation,

- among different combinations of the strain rate hardening and the thermal softening effects applied in the constitutive model, the most noteworthy change in the performance of the target was observed when both were included in the calculations,

- for the flat component layers there was no striking difference in the number of layers required to stop the perforation in the case of rigid and deformable projectiles,

- the ballistic resistance of targets made of various shapes of the component layers was greater for the deformable impactor, and

- the structured component layers are much more efficient than the flat one keeping the overall structure lightweight.

Open Access This article is distributed under the terms of the Creative Commons Attribution 4.0 International License (http:// creativecommons.org/licenses/by/4.0/), which permits unrestricted use, distribution, and reproduction in any medium, provided you give appropriate credit to the original author(s) and the source, provide a link to the Creative Commons license, and indicate if changes were made.

\section{References}

1. Corran, R., Shadbolt, P., Ruiz, C.: Impact loading of plates-an experimental investigation. Int. J. Impact Eng. 1(1), 3-22 (1983)

2. Radin, J., Goldsmith, W.: Normal projectile penetration and perforation of layered targets. Int. J. Impact Eng. 7(2), 229-259 (1988)

3. Almohandes, A., Abdel-Kader, M., Eleiche, A.: Experimental investigation of the ballistic resistance of steel-fiberglass reinforced polyester laminated plates. Compos. Part B Eng. 27(5), 447-458 (1996)

4. Ben-Dor, G., Dubinsky, A., Elperin, T.: On the ballistic resistance of multi-layered targets with air gaps. Int. J. Solids Struct. 35(23), 3097-3103 (1998)

5. Ben-Dor, G., Dubinsky, A., Elperin, T.: Effect of air gaps on ballistic resistance of targets for conical impactors. Theor. Appl. Fract. Mech. 30(3), 243-249 (1998)

6. Ben-Dor, G., Dubinsky, A., Elperin, T.: Effect of air gaps on the ballistic resistance of ductile shields perforated by nonconical impactors. J. Mech. Mater. Struct. 1(2), 279-299 (2006)

7. Woodward, R., Cimpoeru, S.: A study of the perforation of aluminium laminate targets. Int. J. Impact Eng. 21(3), 117-131 (1998)

8. Zukas, J., Scheffler, D.: Impact effects in multilayered plates. Int. J. Solids Struct. 38(19), 3321-3328 (2001)

9. Liang, C.-C., Yang, M.-F., Wu, P.-W., Teng, T.-L.: Resistant performance of perforation of multi-layered targets using an estimation procedure with marine application. Ocean Eng. 32(3-4), 441-468 (2005)

10. Elek, P., Jaramaz, S., Micković, D.: Modeling of perforation of plates and multi-layered metallic targets. Int. J. Solids Struct. 42(3-4), 1209-1224 (2005)

11. Gupta, N., Iqbal, M., Sekhon, G.: Effect of projectile nose shape, impact velocity and target thickness on the deformation behavior of layered plates. Int. J. Solids Struct. 35(1), 37-60 (2008)

12. Dey, S., Børvik, T., Teng, X., Wierzbicki, T., Hopperstad, O.: On the ballistic resistance of double-layered steel plates: an experimental and numerical investigation. Int. J. Solids Struct. 44(20), 6701-6723 (2007)

13. Flores-Johnson, E., Saleh, M., Edwards, L.: Ballistic performance of multi-layered metallic plates impacted by a 7.62-mm APM2 projectile. Int. J. Impact Eng. 38(12), 1022-1032 (2011)

14. Deng, Y., Zhang, W., Cao, Z.: Experimental investigation on the ballistic resistance of monolithic and multi-layered plates against ogival-nosed rigid projectiles impact. Mater. Des. 44, 228-239 (2013)

15. Ben-Dor, G., Dubinsky, A., Elperin, T.: Investigation and optimization of protective properties of metal multi-layered shields: A review. Int. J. Prot. Struct. 3(3), 275-291 (2012)

16. Johnson, G.R., Cook, W.H.: A constitutive model and data for metals subjected to large strains, high strain rates and high temperatures. In: Proceedings of the 7th International Symposium on Ballistics (1983)

17. Johnson, G.R., Cook, W.H.: Fracture characteristics of three metals subjected to various strains, strain rates, temperatures and pressures. Eng. Fract. Mech. 21(1), 31-48 (1985)

18. Lodygowski, T., Rusinek, A., Jankowiak, T., Sumelka, W.: Selected topics of high speed machining analysis. Eng. Trans. 60(1), 69-96 (2012)

19. Sumelka, W., Szajek, K., Łodygowski, T.: Plane strain and plane stress elasticity under fractional continuum mechanics. Arch. Appl. Mech. 89(9), 1527-1544 (2015)

20. Elek, P.M., Jaramaz, S.S., Micković, D.M., Miloradović, N.M.: Experimental and numerical investigation of perforation of thin steel plates by deformable steel penetrators. Thin-Walled Struct. 102, 58-67 (2016)

21. Litoński, J.: Plastic flow of a tube under adiabatic torsion. Bulletin de l'Academie, Polonaise des Sciences Serie des Sciences Techniques XXV(1), 7-14 (1977)

22. Li, H., Wang, X., Duan, J., Liu, J.: A modified Johnson cook model for elevated temperature flow behavior of T24 steel. Mater. Sci. Eng. A 577, 138-146 (2013)

23. Børvik, T., Dey, S., Clausen, A.: Perforation resistance of five different high-strength steel plates subjected to small-arms projectiles. Int. J. Impact Eng. 36(7), 948-964 (2009) 
24. Samantaray, D., Mandal, S., Bhaduri, A.: A comparative study on Johnson-Cook, modified Zerilli-Armstrong and Arrheniustype constitutive models to predict elevated temperature flow behaviour in modified 9Cr-1Mo steel. Comput. Mater. Sci. 47(2), 568-576 (2009)

25. Børvik, T., Hopperstad, O., Berstad, T., Langseth, M.: Numerical simulation of plugging failure in ballistic penetration. Int. J. Solids Struct. 38(34-35), 6241-6264 (2001)

26. Børvik, T., Clausen, A.H., Eriksson, M., Berstad, T., Hopperstad, O.S., Langseth, M.: Experimental and numerical study on the perforation of AA6005-T6 panels. Int. J. Impact Eng. 32(1-4), 35-64 (2005)

27. Holmen, J.K., Børvik, T., Hopperstad, O.S.: Experiments and simulations of empty and sand-filled aluminum alloy panels subjected to ballistic impact. Eng. Struct. 130, 216-228 (2016)

28. Wingrove, A.L.: The influence of projectile geometry on adiabatic shear and target failure. Metall. Trans. 4(8), 1829-1833 (1973)

29. Wilkins, M.L.: Mechanics of penetration and perforation. Int. J. Eng. Sci. 16(11), 793-807 (1978)

30. Børvik, T., Langseth, M., Hopperstad, O., Malo, K.: Perforation of $12 \mathrm{~mm}$ thick steel plates by $20 \mathrm{~mm}$ diameter projectiles with flat, hemispherical and conical noses: part I: experimental study. Int. J. Impact Eng. 27(1), 19-35 (2002)

31. Børvik, T., Hopperstad, O., Berstad, T., Langseth, M.: Perforation of $12 \mathrm{~mm}$ thick steel plates by $20 \mathrm{~mm}$ diameter projectiles with flat, hemispherical and conical noses: part II: numerical simulations. Int. J. Impact Eng. 27(1), 37-64 (2002)

32. Gupta, N., Iqbal, M., Sekhon, G.: Experimental and numerical studies on the behavior of thin aluminum plates subjected to impact by blunt- and hemispherical-nosed projectiles. Int. J. Impact Eng. 32(12), 1921-1944 (2006)

33. Arias, A., Rodríguez-Martínez, J., Rusinek, A.: Numerical simulations of impact behaviour of thin steel plates subjected to cylindrical, conical and hemispherical non-deformable projectiles. Eng. Fract. Mech. 75(6), 1635-1656 (2008)

34. Kpenyigba, K., Jankowiak, T., Rusinek, A., Pesci, R., Wang, B.: Effect of projectile nose shape on ballistic resistance of interstitial-free steel sheets. Int. J. Impact Eng. 79, 83-94 (2015)

35. Rusinek, A., Rodríguez-Martínez, J., Arias, A., Klepaczko, J., López-Puente, J.: Influence of conical projectile diameter on perpendicular impact of thin steel plate. Eng. Fract. Mech. 75(10), 2946-2967 (2008)

36. Iqbal, M., Tiwari, G., Gupta, P., Bhargava, P.: Ballistic performance and energy absorption characteristics of thin aluminium plates. Int. J. Impact Eng. 77, 1-15 (2015)

37. Klepaczko, J.: A practical stress-strain-strain rate-temperature constitutive relation of the power form. J. Mech. Work. Technol. 15(2), 143-165 (1987)

38. Liang, R., Khan, A.S.: A critical review of experimental results and constitutive models for BCC and FCC metals over a wide range of strain rates and temperatures. Int. J. Plast. 15(9), 963-980 (1999)

39. Rusinek, A., Rodríguez-Martínez, J.A., Pesci, R., Capelle, J.: Experimental characterisation and modelling of the thermoviscoplastic behaviour of steel AISI 304 within wide ranges of strain rate at room temperature. J. Theor. Appl. Mech. 48(4), 1027-1042 (2010)

40. Pothnis, J., Perla, Y., Arya, H., Naik, N.: High strain rate tensile behavior of aluminum alloy 7075 T651 and IS 2062 mild steel. J. Eng. Mater. Technol. Trans. ASME 133(2), 021026 (2011)

41. Hor, A., Morel, F., Lebrun, J.-L., Germain, G.: An experimental investigation of the behaviour of steels over large temperature and strain rate ranges. Int. J. Mech. Sci. 67, 108-122 (2013)

42. Su, X., Wang, G., Li, J., Rong, Y.: Dynamic mechanical response and a constitutive model of Fe-based high temperature alloy at high temperatures and strain rates. SpringerPlus 5(1), 504 (2016)

43. 43. Szymczyk, M., Sumelka, W., Ldygowski, T.: Designing of multi layered protective panels against improvised debris. In: Rusinski, E., Pietrusiak, D. (eds.) Proceedings of the 13th International Scientific Conference, pp. 561-570. Springer International Publishing, Lecturer Notes in Mechanical Engineering (2016). doi:10.1007/978-3-319-50938-9_58

44. Corbett, B.: Numerical simulations of target hole diameters for hypervelocity impacts into elevated and room temperature bumpers. Int. J. Impact Eng. 33(1-12), 431-440 (2006)

45. Schwer, L.: Aluminium plate perforation: a comparative case study using lagrange with erosion, multi-material ale, and smooth particle hydrodynamics. In: 7th European LS-DYNA Conference (2009) 